\title{
La comunicación remota en hospitales en tiempos de COVID-19
}

\section{Remote communication in hospitals during times of COVID-19}

$E^{n}$ n la literatura científica, existen múltiples definiciones del significado del término "telemática en salud", "comunicación remota" o "telesalud". Una de las más extendidas, y creo que más adecuada, es la proporcionada por la Organización Mundial de la Salud (World Health Organization [WHO], 1998, p. 14) en la que se define como:

La prestación de servicios de salud, por parte de todos los profesionales sanitarios con la ayuda de las tecnologías de la información y la comunicación (TICS) para el intercambio de información válida. Además, el uso de la comunicación remota permite el diagnóstico, tratamiento, prevención de enfermedades y lesiones, investigación, evaluación y la educación continua de los proveedores de atención sanitaria, todo en el interés de promover la salud de las personas y su entorno.

A medida que ha ido avanzando el siglo XXI y el enorme progreso experimentado por estas tecnologías, ha fomentado que un número creciente de hospitales hayan optado por utilizar las historias clínicas electrónicas. En este caso, los profesionales sanitarios encargados de atender sus consultas en la modalidad remota de seguimiento tenían la gran responsabilidad de proteger las historias clínicas de los pacientes y mantener la confidencialidad de la información sobre sus tratamientos (Eze, Mateus, \& Cravo Oliveira Hashiguchi, 2020; Gruska, Aigner, \& Altenberger, 2020). Previamente, la comunicación remota en hospitales se había utilizado para promover el autocuidado a través de la monitorización de enfermedades crónicas, con el fin de facilitar la asistencia virtual a las consultas a aquellos pacientes que no podían asistir de manera física, mejorando de esta manera la atención al paciente.

Una de las ventajas clave de la comunicación remota hospitalaria es su capacidad para aumentar el acceso a la atención sanitaria, al ofrecer a los pacientes y a sus cuidadores la oportunidad de recibir atención en sus hogares (Berman, \& Fenaughty. 2005; Delgoshaei, Mobinizadeh, \& Mojdekar, 2017; Eze, 2020). Con la aparición y expansión de la COVID-19, este aspecto se ha vuelto más importante puesto que tanto los pacientes como los trabajadores de la salud se han visto en la tesitura creciente de adoptar nuevas formas de atención sanitaria, pero con un contacto físico mínimo (Eze, 2020).

Los servicios sanitarios ofertados en el ámbito de las TICs se están implementando de manera creciente en diferentes áreas clínicas como la cardiología, dermatología, rehabilitación, etc. En estos últimos 10 años, se ha incrementado el número de estudios centrados en el análisis de la eficiencia económica, calidad de vida relacionada con la salud, satisfacción y experiencias vividas por los pacientes y/o sus cuidadores, además de tendencias futuras de la telesalud (Catalán-Matamoros, \& López-Villegas, 2015). De entre estos estudios, cabe destacar los estudios PONIENTE Y NORDLAND (López-Villegas, CatalánMatamoros, \& RoblesMusso, 2016; Lopez-Villegas, Catalan-Matamoros, \& Peiro, 2020; Bautista-Mesa, LopezVillegas, \& Peiro, 2020), centrados en el análisis a medio y largo plazo de la comunicación remota de los marcapasos cardíacos. Los resultados de estos estudios han demostrado que desde la perspectiva del Sistema Sanitario Público de Salud, se trata de una tecnología fiable en cuanto a la transmisión de información, más eficiente que el seguimiento convencional puesto que se reduce el número de visitas físicas programadas al hospital, disminuyen la carga de trabajo y los costes asociados, se incrementa la satisfacción experimentada por los pacientes, además es una tecnología segura porque en caso de ser necesario permite a los sanitarios actuar de forma precoz ante alteraciones cardiovasculares o técnicas del dispositivo que pudieran comprometer la vida de los pacientes. Y desde la perspectiva del paciente, los costes informales también se reducen de manera significativa, lo que supone un alivio tanto para los pacientes como para sus cuidadores cuando tienen que enfrentarse a condiciones de salud difíciles que les imposibiliten o dificulten la movilidad (Lopez-Villegas, Leal-Costa, \& Perez-Heredia, 2021). 
Con la llegada de la pandemia provocada por la expansión de la enfermedad de la COVID-19, se adoptaron medidas que incluyeron la limitación de la libre circulación de ciudadanos para evitar la propagación del virus, no saturar el sistema sanitario y poder centrarse en dar la mejor atención sanitaria posible a los pacientes afectados por la COVID-19. Esta situación provocó una reducción de la actividad asistencial habitual tanto en los centros de Atención Primaria como en los hospitales. La saturación del sistema sanitario que estuvo al borde del colapso en la primavera

Servicios sanitarios de manera remota y tecnologías de telesalud: parte integral de un número importante de centros hospitalarios durante la pandemia de coronavirus

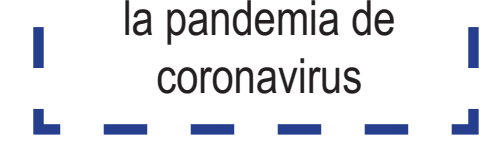

del año 2020, implicó una rápida reestructuración de los procesos asistenciales, así como una reubicación de los recursos físicos y materiales. Esta situación precipitó que la implementación de la comunicación remota en los hospitales, haya sido más rápida que si no hubiera aparecido la COVID-19 en 」 nuestras vidas, especialmente en los países del primer mundo. En estos momentos, la comunicación remota en el ámbito hospitalario, va más allá del teléfono y el correo electrónico, las videoconferencias y/o los portales webs, ahora incluye una variedad cada vez mayor de nuevas TICs que utilizan video bidireccional, teléfonos inteligentes, herramientas inalámbricas, APPs, etc. (Catalán-Matamoros, 2016).

A pesar de haberse demostrado que la comunicación remota llevada a cabo en los centros hospitalarios ofrece beneficios tanto en costes como en calidad de vida a pacientes, cuidadores y a los diferentes sistemas públicos de salud, son muchos los gobiernos que en la mayor parte de las regiones del mundo todavía no se han decidido por implementar estas tecnologías en sus países debido principalmente a problemas económicos, infraestructuras, desarrollo tecnológico, voluntad, etc.

Finalmente, me gustaría comentar que la pandemia provocada por el SARS-CoV-2 y la mejora de la comunicación entre los profesionales sanitarios, pacientes y/o sus cuidadores inevitablemente ha generado que los servicios sanitarios proporcionados de manera remota y las tecnologías asociadas a la telesalud se hayan transformado en una parte integral de un número importante de centros hospitalarios. Independientemente, de los obstáculos existentes o que puedan surgir en el futuro, está claro que la comunicación remota en el ámbito hospitalario está aquí para quedarse.

\section{Referencias bibliográficas}

Bautista-Mesa, R. J., Lopez-Villegas, A., Peiro, S., Catalan-Matamoros, D., Robles-Musso, E., LopezLiria, R., \& Leal-Costa, C. (2020). Long-term costutility analysis of remote monitoring of older patients with pacemakers: the PONIENTE study. BMC Geriatrics, 20(1), 474.

Berman, M., \& Fenaughty, A. (2005). Technology and managed care: patient benefits of telemedicine in a rural health care network. Health Economics, 14(6), 559-573.

Catalán-Matamoros, D., \& López-Villegas, A. (2015). Telemedicine in the Artic: communication challenges. Revista Española de Comunicación en Salud, $6(1), 1-2$.

Delgoshaei, B., Mobinizadeh, M., Mojdekar, R., Afzal, E., Arabloo, J., \& Mohamadi, E. (2017). Telemedicine: A systematic review of economic evaluations. Medical Journal of the Islamic Republic of Iran, 31, 113.

Eze, N. D., Mateus, C., \& Cravo Oliveira Hashiguchi, T. (2020). Telemedicine in the OECD: An umbrella review of clinical and cost-effectiveness, patient experience and implementation. PloS One, 15(8), e0237585.

Gruska, M., Aigner, G., Altenberger, J., Burkart-Küttner, D., Fiedler, L., Gwechenberger, M., ... Working Group Rhythmology of the Austrian Cardiological Society (2020). Recommendations on the utilization of telemedicine in cardiology. Wiener Klinische Wochenschrift, 132(23-24), 782-800.

López-Villegas, A., Catalán-Matamoros, D., RoblesMusso, E., \& Peiró, S. (2016). Workload, time and costs of the informal cares in patients with telemonitoring of pacemakers: the PONIENTE study. Clinical Research in Cardiology: Official Journal of the German Cardiac Society, 105(4), 307-313. 
Lopez-Villegas, A., Catalan-Matamoros, D., Peiro, S., Lappegard, K. T., \& Lopez-Liria, R. (2020). Costutility analysis of telemonitoring versus conventional hospital-based follow-up of patients with pacemakers. The NORDLAND randomized clinical trial. PloS One, 15(1), e0226188.

Lopez-Villegas, A., Leal-Costa, C., Perez-Heredia, M., Villegas-Tripiana, I., \& Catalán-Matamoros, D. (2021). Knowledge Update on the Economic Evaluation of Pacemaker Telemonitoring Systems. International Journal of Environmental Research and Public Health, 18(22), 12120.

World Health Organization. (1998). Health telematics policy in support of WHO's Health-For-All strategy for global health development. Report of the WHO Group Consultation on Health Telematics, 11-16. Recuperado de: https://apps.who.int/iris/bitstream/ handle/10665/63857/WHO_DGO_98.1.pdf?sequenc e=1\&isAllowed=y

\section{Antonio López-Villegas}

Unidad de Investigación, Hospital de Poniente, El EjidoAlmería, España.

antoniolopezvillegas@andaluciajunta.es 